\title{
An Adaptive Spectral Kurtosis Method Based on Optimal Filter
}

\author{
Yanli Yang and Ting Yu \\ Tianjin Key Laboratory of Optoelectronic Detection Technology and Systems, Tianjin Polytechnic University, Tianjin 300387, China
}

Correspondence should be addressed to Yanli Yang; yyl070805@163.com

Received 1 June 2017; Revised 29 September 2017; Accepted 8 October 2017; Published 5 November 2017

Academic Editor: Carlo Rainieri

Copyright (C) 2017 Yanli Yang and Ting Yu. This is an open access article distributed under the Creative Commons Attribution License, which permits unrestricted use, distribution, and reproduction in any medium, provided the original work is properly cited.

\begin{abstract}
As a useful tool to detect protrusion buried in signals, kurtosis has a wide application in engineering, for example, in bearing fault diagnosis. Spectral kurtosis (SK) can further indicate the presence of a series of transients and their locations in the frequency domain. The factors influencing kurtosis values are first analyzed, leading to the conclusion that amplitude, not the frequency of signals, and noise make major contribution to kurtosis values. It is helpful to detect impulsive components if the components with big amplitude are removed from composite signals. Based on this cognition, an adaptive SK algorithm is proposed in this paper. The core steps of the proposed SK algorithm are to find maxima, add window around maxima, merge windows in the frequency domain, and then filter signals according to the merged window in the time domain. The parameters of the proposed SK algorithm are varying adaptively with signals. Some experimental results are presented to demonstrate the effectiveness of the proposed algorithm.
\end{abstract}

\section{Introduction}

Kurtosis proposed by Dyer and Stewart in the 1970s [1] is used generally in the statistical field to describe the distribution, or skewness, of observed data around the mean. In engineering, kurtosis can be used to detect machine faults. It is already used for diagnosis of bearing, because it helps to find the crack failure from vibration signals. As a dimensionless parameter, kurtosis index has nothing to do with the bearing size, speed, and load, but it is sensitive to impact signals. It is especially suitable for the surface damage fault detection in the early fault diagnosis.

However, kurtosis is a global method and it only provides a vague suggestion. To solve this problem, spectral kurtosis (SK) is introduced by Dwyer [2] for detecting impulsive events in sonar signals. A formalization of SK is given by Antoni [3], in which the short-time Fourier transform(STFT-) based SK is proposed. At the same time, Antoni and Randall [4] proposed the concept of kurtogram which uses the SK as a basis for detecting ad hoc detection filters to extract mechanical signature of faults. The SK is a statistical tool that can indicate the presence of series of transients and their locations in the frequency domain [3]. It provides a robust way of detecting incipient faults even in the presence of strong masking noise and offers an almost unique way of designing optimal filters for filtering out the mechanical signature of faults, which served as a useful tool to monitor the running status and diagnosis fault of mechanical machine [4]. The maximum correlation kurtosis deconvolution technique [5], the Morlet wavelet [6], and the probabilistic principal component analysis [7] can be used to enhance the capability of the SK.

Although the SK has attracted much attention due to the kurtogram, it is pointed out in [8] that the kurtogram cannot cope well with signals of composite and frequency of randomly impulsive nature, and it is vulnerable to random extraneous signals. It is further pointed out in [9] that the STFT-based SK technique may not be practical because it is unrealistic to find the optimal bandwidth and frequency of filters by examining all the window lengths. To overcome the limitation of kurtogram, a method for the selection of optimal bandpass filter to calculate SK, termed as protrugram, is proposed in [8]. An improved kurtogram was proposed in [10] based on wavelet packet. It is an intrinsic demand of the SK to separate the impulsive components into subband signals adaptively and then calculate the value of kurtosis. In fact, an adaptive windowed SK is proposed by Wang and Liang [9] through merging windows along the frequency 
axis in the frequency domain, which can optimize filter bandwidth and locate center frequency when used in fault detection on rotate machine. It is shown in [11] that the adaptive windowed SK more effectively extracts signatures of multiple bearing faults. Nevertheless, the optimal merging window associated with the highest SK does not match better with the signal transient feature when the transient impulse decays slowly [12]. An adaptive SK filtering method based on the Morlet wavelet is proposed in [12] to extract the signal transient buried in noise. A kurtosis-guided adaptive demodulation technique based on the tunable- $\mathrm{Q}$ wavelet transform is proposed in [13] for bearing faults detection. An optimized SK is proposed in [14] for selecting the best demodulation band to extract bearing fault-related impulsive content from vibration signals contaminated with strong electromagnetic interference.

The SK method is already proven to be useful in detecting nonstationary components of signals. Empirical mode decomposition (EMD) is a method to deal with nonstationary signals adaptively [15]. Inspired by our previous work $[15,16]$ and the work done in [8-14], an adaptive SK algorithm is proposed in this paper. In Section 2, kurtosis and SK are reviewed briefly. Section 3 analyzes the factors that have an influence on kurtosis values. In Section 4, the detailed description of the proposed adaptive SK algorithm is presented. Then, Section 5 shows some simulated examples. Finally, the conclusions are drawn in Section 6.

\section{Kurtosis and Spectral Kurtosis}

Kurtosis is a reflection of the probability distribution of signals. Mathematically, for a time series $x(t)$, kurtosis is defined as [17]

$$
K=\frac{E\left\{(x-\mu)^{4}\right\}}{\left[E\left\{(x-\mu)^{2}\right\}\right]^{2}}=\frac{\mu_{4}}{\sigma^{4}},
$$

where $\mu$ is the mean of $x(t), \sigma$ is the standard deviation of $x(t)$, and $E\{\cdot\}$ is the expectation operator. Equation (1) shows that kurtosis is the fourth central moment divided by the square of the variance. The kurtosis of the normal distribution is 3 , so some definitions of kurtosis subtract 3 from the computed value. In this paper, (1) is used to calculate kurtosis in the subsequent analysis.

By using the theory of signal and system, Antoni [3] has given the definition of SK as the fourth-order spectral cumulant. For a signal $x(t)$, the SK is written as [3]

$$
K_{x}(f)=\frac{\left\langle X^{4}(t, f)\right\rangle}{\left\langle X^{2}(t, f)\right\rangle^{2}}-2,
$$

where $K_{x}(f)$ denotes the SK of $x(t), X^{2}(t, f)$ represents the time/frequency envelope of the signal $x(t)$, and $\langle\cdot\rangle$ stands for the time averaging operator. For the case of detecting a signal $x(t)$ buried in strong additive noise $n(t)$, written as

$$
y(t)=x(t)+n(t),
$$

the SK of $y(t)$ can be expressed by [4]

$$
K_{y}(f)=\frac{K_{x}(f)}{[1+\rho(f)]^{2}},
$$

where $K_{y}(f)$ denotes the SK of $y(t)$ and $\rho(f)$ is the noise-tosignal ratio (NSR).

The SK is an effective and important tool to locate the frequency bands with a high amount of impulsiveness. However, the biggest value of kurtosis does not locate at the lowest level in kurtogram. Here, a simple example is presented to illustrate it. The signal considered is

$$
y(t)=p(t)+x(t),
$$

where $x(t)$ is a strong signal and $p(t)$ is a periodic impulsive signal, as shown in Figure 1. According to [18], the signal $p(t)$ shown in Figure 1 is a simulated vibration signal arising from a rolling element bearing which can be written as

$$
\begin{gathered}
p(t)=\sum_{i} A_{i} s_{i}\left(t-i T_{p}-\tau_{i}\right)=\sum_{i} A_{i} e^{-\beta\left(t-i T_{p}-\tau_{i}\right)} \sin \omega_{r} \\
\cdot\left(t-i T_{p}-\tau_{i}\right) u\left(t-i T_{p}-\tau_{i}\right),
\end{gathered}
$$

where $A_{i}$ is the amplitude of the fault impulse, $\beta$ denotes the structural damping characteristic, $T_{p}$ denotes the time period corresponding to the fault characteristic frequency, $\tau_{i}$ represents the effect of random slippage of the rollers, $\omega_{r}$ denotes the excited resonance frequency, and $u(t)$ is a unit step function. In (5), $x(t)$ are some harmonic interferences which can be written as [13]

$$
x(t)=\sum_{l} A_{l} \sin 2 \pi f_{l} t .
$$

Combining (5), (6), and (7), the signal can be expressed as

$$
\begin{aligned}
& y(t)=p(t)+x(t)=\sum_{i} A_{i} e^{-\beta\left(t-i T_{p}-\tau_{i}\right)} \sin \omega_{r} \\
& \cdot\left(t-i T_{p}-\tau_{i}\right) u\left(t-i T_{p}-\tau_{i}\right)+\sum_{l} A_{l} \sin \left(2 \pi f_{l} t\right) .
\end{aligned}
$$

We can see from Figure 1 that the center of impulsive signal is located at $1500 \mathrm{~Hz}$. The kurtogram results of the simulated signal are shown in Figure 2. It is clear from Figure 2(a) that the biggest value of kurtosis is located on level 2, and the band is located between $5000 \mathrm{~Hz}$ and $7500 \mathrm{~Hz}$. However, Figure 2(b) shows that the biggest value of kurtosis is located on level 5, but it is less than 3.2. Some questions naturally arise. The first question is as follows: why is the value of kurtosis big for a signal but small in its subbands? Another question is why does the SK value drop obviously in the case of strong noise? To answer those questions, we argue that the main reasons of influencing on the value of kurtosis must be clear. In the next section, we discuss the factors that have an influence on kurtosis values. 


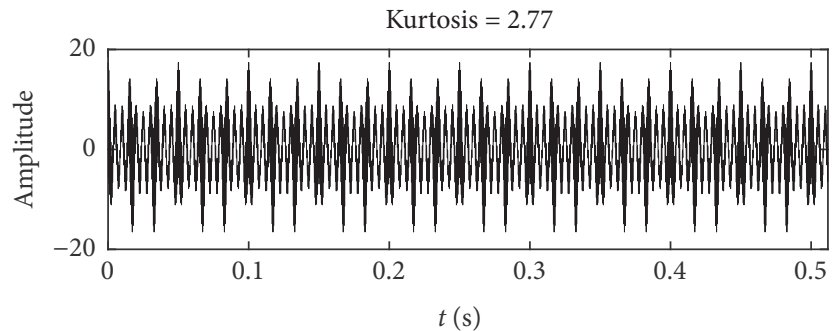

(a)

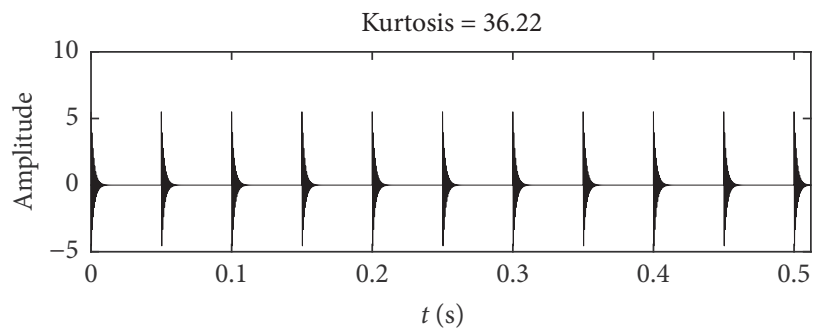

(c)

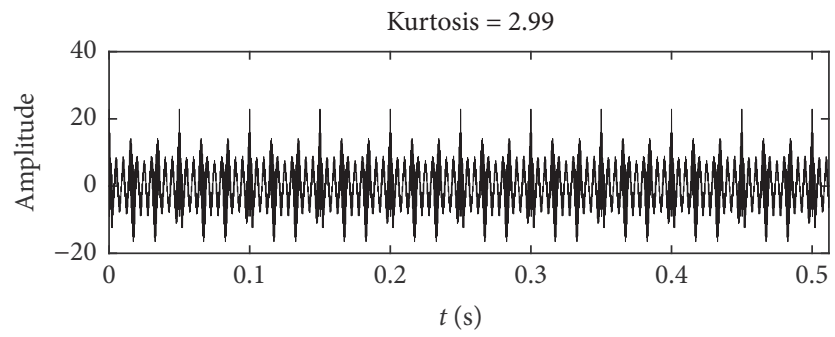

(e)

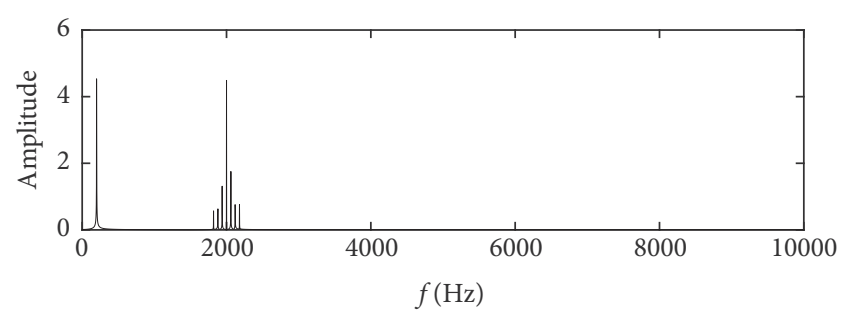

(b)

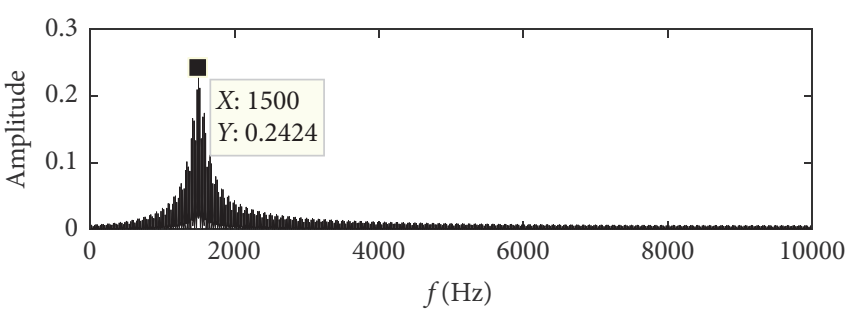

(d)

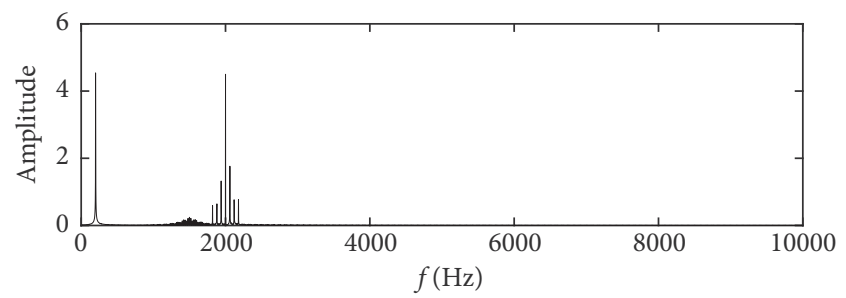

(f)

Figure 1: The signal and its spectrum. (a) is the signal, (c) is the impulsive signal, and (e) is the signal added with the impulsive signal. (b), (d), and (f) are the spectrum of corresponding (a), (c), and (e).

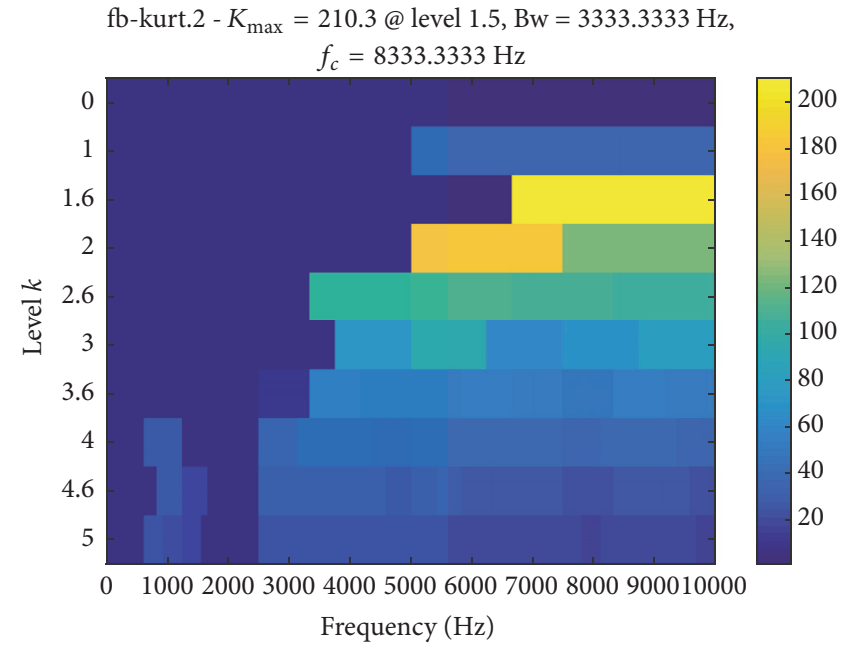

(a)

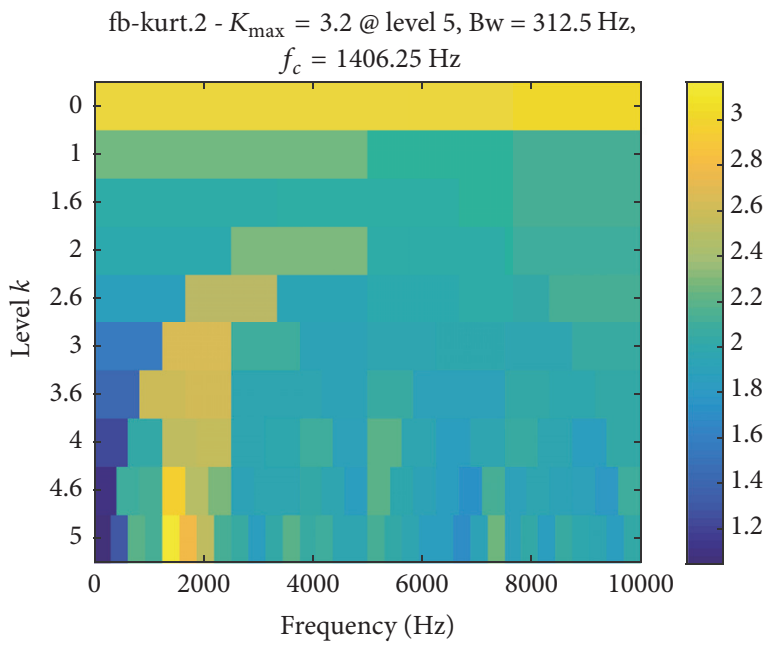

(b)

FIGURE 2: The kurtogram results of the signal shown in Figure 1. (a) is the result of the signal without noise. (b) is the result of the signal with additive noise, where the signal-to-noise ratio (SNR) is -10 . 

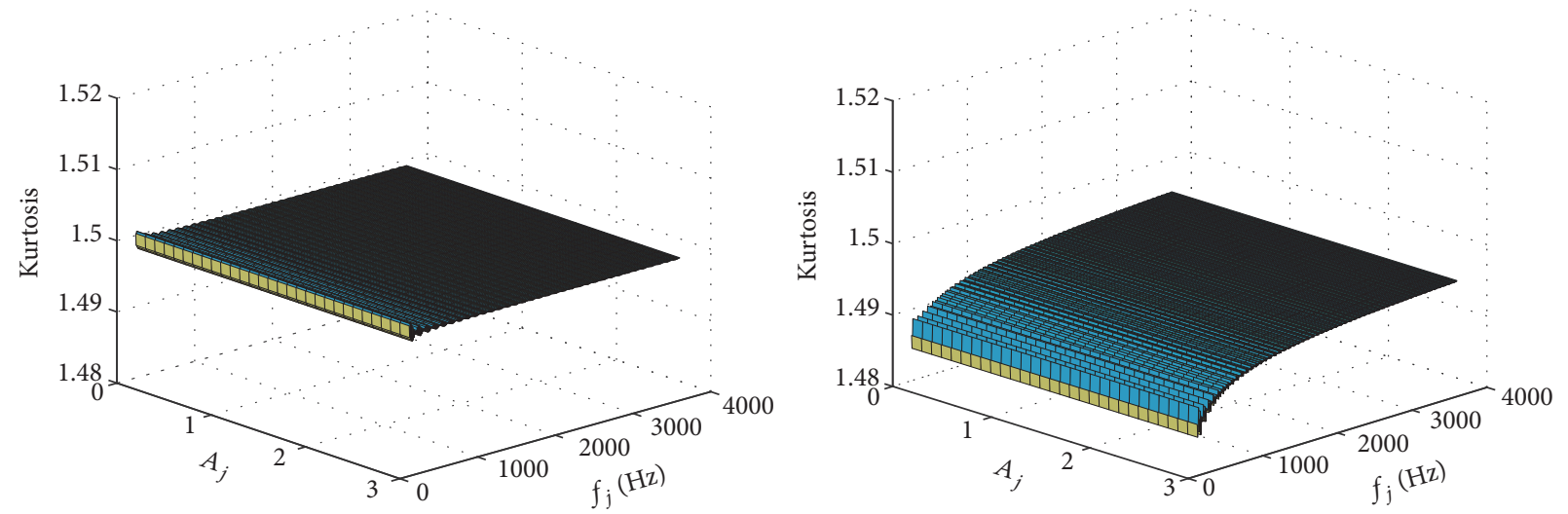

FIGURE 3: Illustration of kurtosis of noncomposite signals.

\section{The Factors Influencing Kurtosis Values}

It is well known that kurtosis is sensitive to impulse signals, which can be used to evaluate the strength of shock components buried in signals. However, the value of kurtosis can be influenced by noise. Besides noise, amplitude of signals is another factor to have an effect on the value of kurtosis. Detailed analysis of the factors, amplitude, frequency, and noise, affecting kurtosis is presented as follows.

3.1. Kurtosis of Noncomposite Signals. We consider a singlefrequency signal that is written as

$$
x_{j}(t)=A_{j} \cos \left(2 \pi f_{j} t\right)
$$

and a chirp signal that is written as

$$
s_{j}(t)=A_{j} \cos \left(2 \pi f_{j} t^{2}\right) .
$$

The kurtosis values of the signals expressed by (9) and (10) are shown in Figure 3. It is clear from this figure that the amplitude and frequency have a small effect on the values of kurtosis for noncomposite signals.

3.2. Kurtosis of Composite Signals. It is shown in [8] that a protrusion in signals will cause a big kurtosis value. We consider the impulsive signal expressed by (6) to analyze the changing rule of kurtosis values. The kurtosis values are shown in Figure 4 for different number of impulses. For the signals with a single impulse, the kurtosis value is very high, just as shown in Figure 4. It can also be seen from this figure that the kurtosis value decreases with increasing the number of impulses.

We then consider the impulsive signal expressed by (6) added by a sine signal, which is written as

$$
\begin{aligned}
& y(t)=p(t)+x_{j}(t)=\sum_{i} A_{i} e^{-\beta\left(t-i T_{p}-\tau_{i}\right)} \sin \omega_{r} \\
& \cdot\left(t-i T_{p}-\tau_{i}\right) u\left(t-i T_{p}-\tau_{i}\right)+A_{j} \sin \left(2 \pi f_{j} t\right) .
\end{aligned}
$$

The kurtosis values of $y(t)$ for different amplitude $A_{j}$ and frequency $f_{j}$ of $x_{j}(t)$ are shown in Figure 5. It is clear from this figure that the amplitude of $x_{j}(t)$ is the major influencing factor on kurtosis values. The kurtosis value becomes smaller as the amplitude of $x_{j}(t)$ increases. Hence, we get that the greater the harmonic interference is the smaller the kurtosis values are.

Consider the impulsive signal expressed by (6) added with a chirp signal, which is written as

$$
\begin{aligned}
& y(t)=p(t)+s_{j}(t)=\sum_{i} A_{i} e^{-\beta\left(t-i T_{p}-\tau_{i}\right)} \sin \omega_{r} \\
& \cdot\left(t-i T_{p}-\tau_{i}\right) u\left(t-i T_{p}-\tau_{i}\right)+A_{j} \sin \left(2 \pi f_{j} t^{2}\right) .
\end{aligned}
$$

The kurtosis values are shown in Figure 6, where $f_{j}=1$. We can see that the kurtosis values decrease gradually from 18.97 to 2.303 with $A_{j}$ increasing from 0 to 5 . Figure 6(b) shows that the kurtosis value becomes very small because the impulsive signal only has a slight move. Hence, we get that the amplitude of $s_{j}(t)$ has a big influence on the kurtosis values.

3.3. Influence of SNR. We consider an impulsive signal that is shown in the lower-right corner of Figure 4 . The kurtosis value is 18.97 for the impulsive signal without noise. Figure 7 shows that the kurtosis value increases gradually from 3.001 to 18.95 with SNR increasing from -20 to 30 . However, it increases rapidly from 3.585 to 17.57 with SNR increasing from -10 to 10 . Hence, we can get that SNR has a great effect on the value of kurtosis. Figure 7 implies that SNR needs to be greater than -9 to detect the impulsive signal if it is assumed that it can be detected when the kurtosis value is bigger than 4.

\section{An Adaptive SK Algorithm}

From the previous section, we learn that amplitude, not the frequency of signals, and noise make major contribution to kurtosis values. It is possible to detect the impulsive components easily from a composite signal, if we can remove the components that have large amplitude. Fortunately, it is easy 


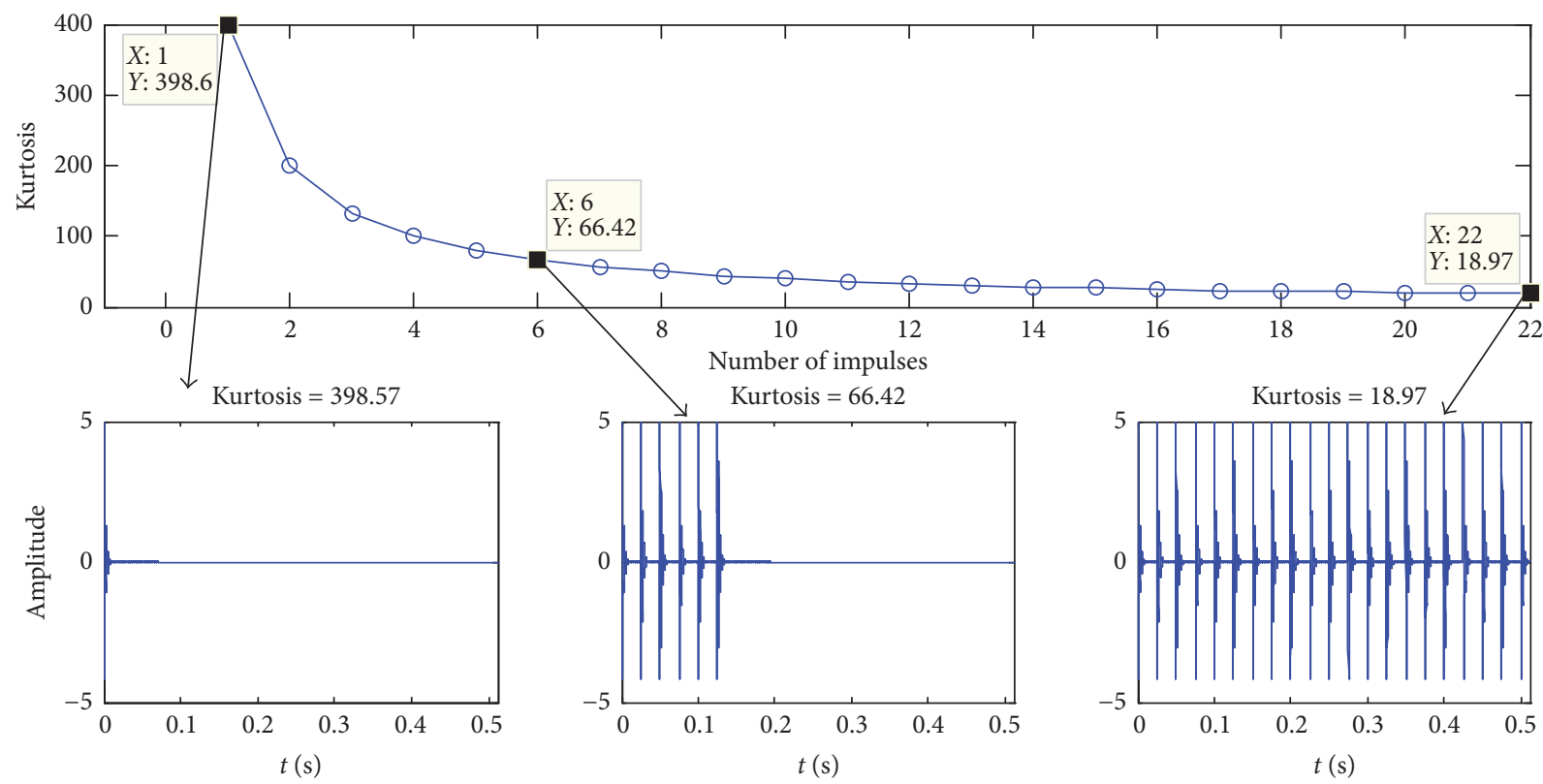

FIGURE 4: Illustration of kurtosis values for different number of impulses.

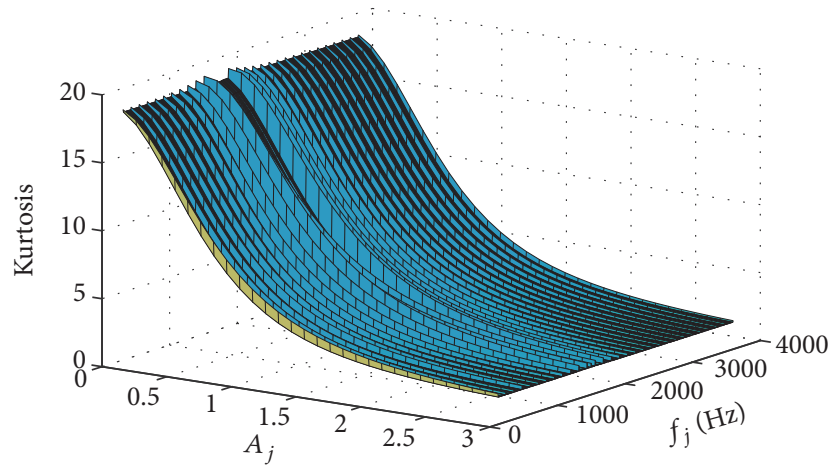

FigURE 5: Illustration of the kurtosis values of the signal expressed by (11).

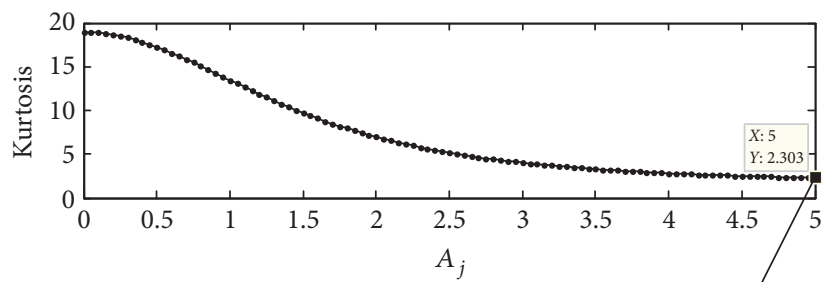

(a)

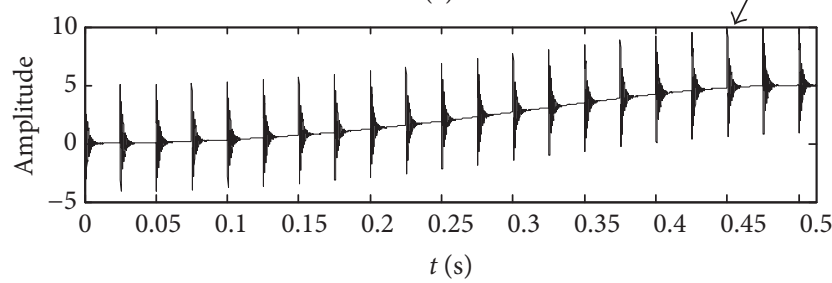

(b)

FIGURE 6: Illustration of kurtosis values of the signal expressed by (12). (a) The kurtosis value varying with $A_{j}$. (b) A signal waveform.

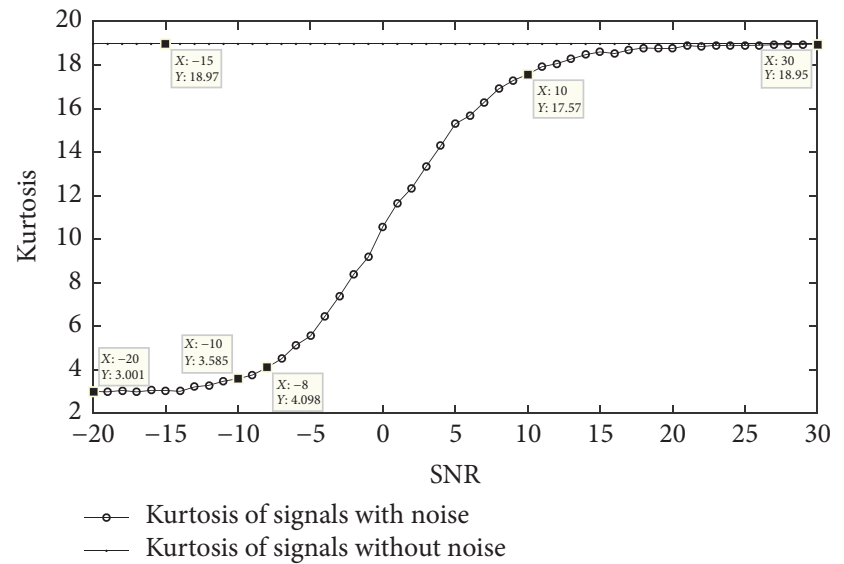

FIGURE 7: Illustration of the influence of SNR on kurtosis values.

to get the amplitude of components from spectrum of signals. Based on this cognition, we propose an adaptive SK algorithm. The flow chart showing the implementation of the proposed adaptive SK algorithm is shown in Figure 8.

As shown in Figure 8, the proposed SK algorithm has three key steps. The first key step is to find out the maxima points in the frequency domain. A time signal is transformed to the frequency domain by using fast Fourier transform (FFT). Then, it is easy to find all the local maximum points in the amplitude spectrum. There is no doubt that many local maximum points will be marked out especially for composite signals with some noise. According to the analysis in the previous section, the components with large amplitude need to be removed. Hence, it is advisable to select a number of local maxima, the value of which is big enough to deal with. Although many SK methods are also based on FFT, such 


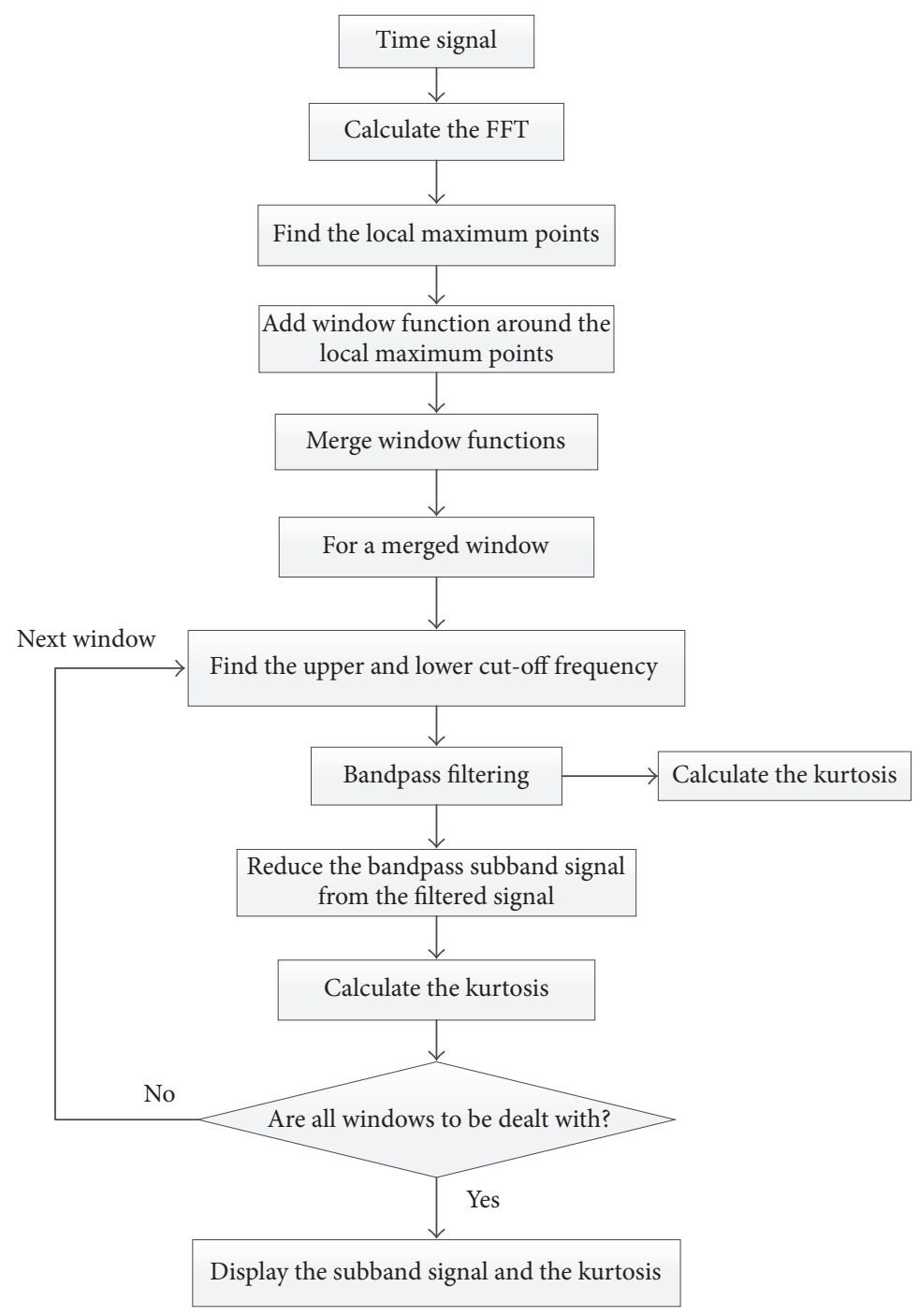

FIGURE 8: The framework of the proposed adaptive SK.

as kurtogram, using maximum points to obtain SK is our characteristic.

Another key step is to add window functions around each of the marked maximum points and merge windows in the frequency domain. The window is used to determine the cutoff frequency of filters. If the window is set too small, the filter has a very narrow bandwidth. The window length $l_{w}$ is calculated by

$$
l_{w}=\beta d_{m},
$$

where $d_{m}$ denotes the mean distance of the local maxima and $\beta$ is a constant. Too narrow bandwidth of filter will cause the filtered subband signal to have some distortion which can cause a large kurtosis value (an example is shown in Figure 9). Hence, we need to merge the windows added around local maximum points. The windows are merged into a bigger one if they are overlapped. On the other hand, the fewer number of windows, the faster running speed of the proposed algorithm.
The third key step is to filter the signal according to the merged window in the time domain. The upper and lower cut-off frequencies of a bandpass filter are calculated according to the merged window. Then, the signal is filtered by using the constructed bandpass filter. The kurtosis value of the subband signal obtained by bandpass filtering is calculated. Subtract the subband signal from the filtered signal to obtain kurtosis value of another subband signal.

Here, an example is presented to illustrate the procedure of the proposed adaptive SK method, as shown in Figure 10. We can see from Figure 10(a) that there are many local maximum points in the spectrum of the signal. Thirty local maximum points, the value of which is big enough, are selected out, as shown in Figure 10(b). A window function is added around each selected local maximum point, as shown in Figure 10(c). It can be clearly seen that some window functions are overlapped. Those overlapped windows are merged into a bigger one. As shown in Figure 10(d), there are only three windows. Then, we can filter the signal by using three bandpass filters and calculate its kurtosis value. 


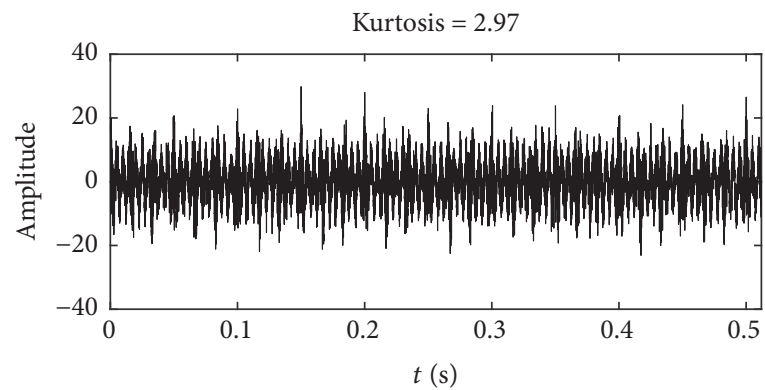

(a)

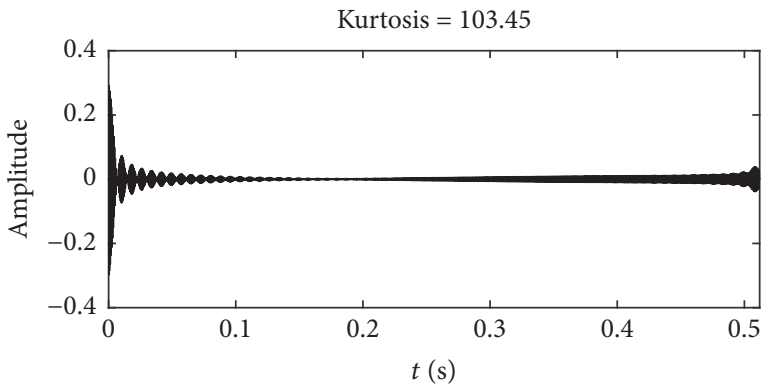

(c)

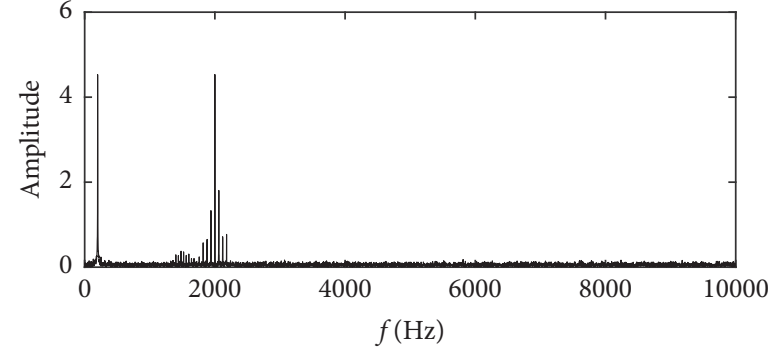

(b)

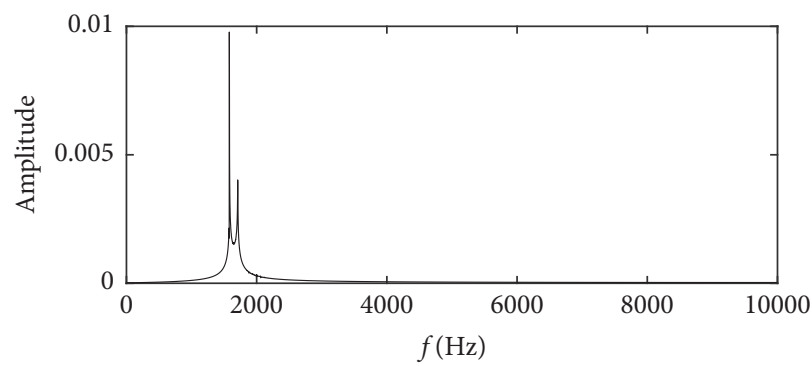

(d)

FIGURE 9: An example to illustrate the large kurtosis value of a subband signal filtered by a narrow bandwidth filter. (a) is the original signal and (c) is the subband signal filtered by a narrow bandwidth filter. (b) and (d) are the spectrum of corresponding (a) and (c).

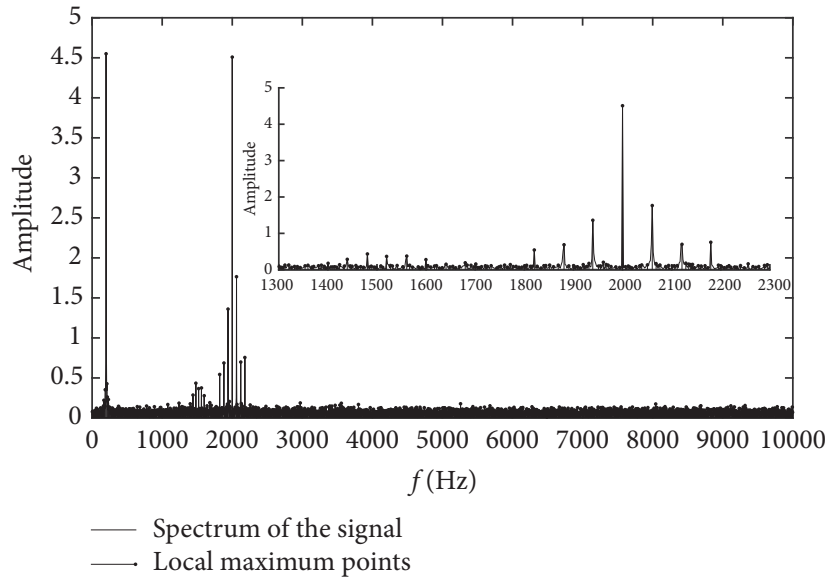

(a)

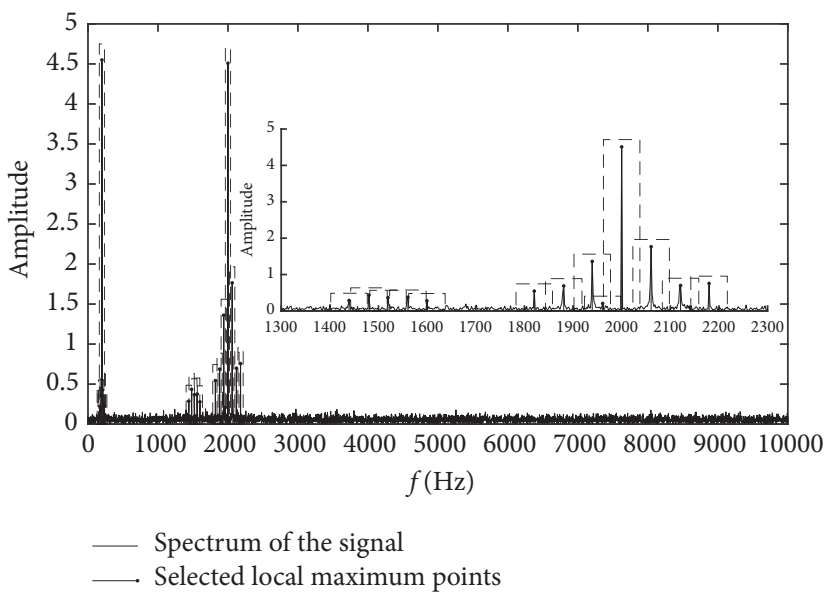

(c)

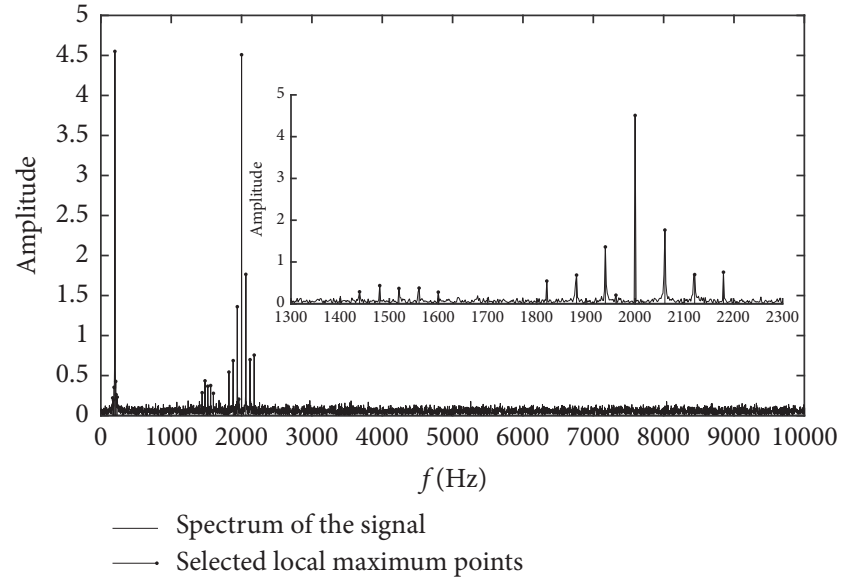

(b)

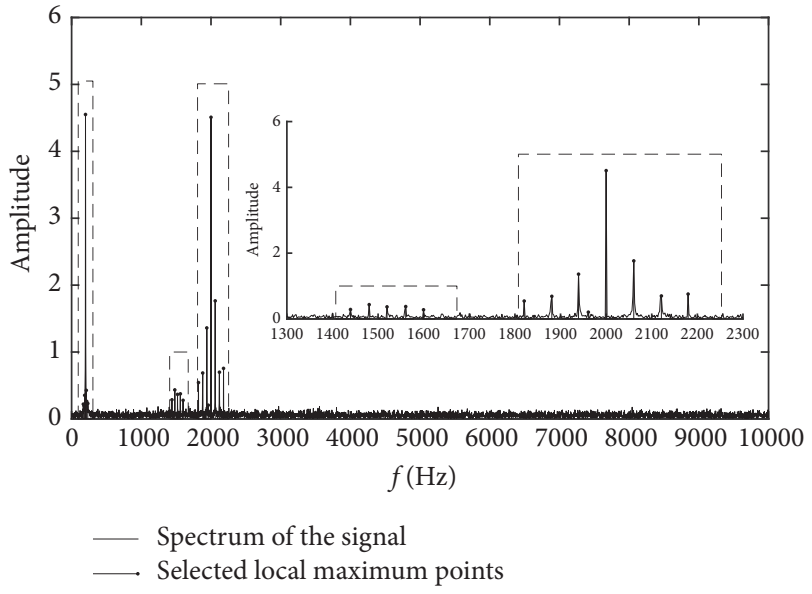

(d)

Figure 10: An example to illustrate the proposed adaptive SK method. (a) The spectrum of a signal and its local maxima. (b) The selected local maximum points. (c) The windows added around the selected local maximum points. (d) Merged window functions. 
TABLE 1: The kurtosis values of the proposed method and the original signals.

\begin{tabular}{|c|c|c|c|c|c|c|c|c|c|c|}
\hline SNR & -20 & -19 & -18 & -17 & -16 & -15 & -14 & -13 & -12 & -11 \\
\hline Kurtosis of the proposed method & 3.64 & 3.95 & 3.46 & 4.37 & 4.50 & 4.91 & 5.34 & 6.09 & 6.80 & 7.68 \\
\hline Kurtosis of the original signals & 2.96 & 3.07 & 2.96 & 2.96 & 3.02 & 3.04 & 3.13 & 3.14 & 3.21 & 3.39 \\
\hline
\end{tabular}

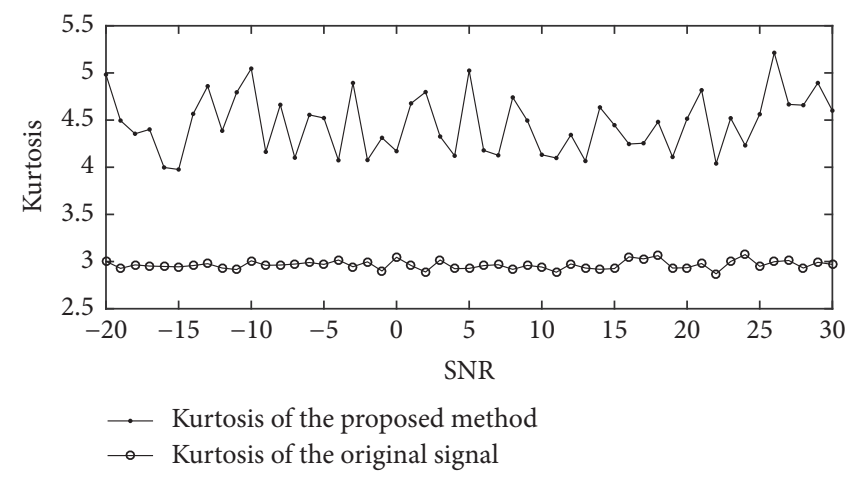

FIGURE 11: The kurtosis values of the proposed method and the original signal for the simulated signals with different SNR.

\section{Simulation Analysis}

To testify the correctness and effectiveness of the proposed adaptive SK algorithm, simulation analysis on the simulated vibration signal expressed by (8) is conducted. The simulation signal is shown in Figure 1. We select the biggest kurtosis value generated by the proposed algorithm for comparison with the kurtosis of the original signal. The comparison result for different SNR is shown in Figure 11. This figure shows that the kurtosis of the proposed method is changing around 4.5 with different SNR, while the kurtosis of the original signals is changing around 3. It implies that the proposed method works stably even in very low SNR case. Hence, we can get that the proposed method can be used to detect the impulsive signal.

For comparison with the kurtogram result shown in Figure 2, a detailed signal expressed by (8), the SNR of which is -10 , is selected to calculate the SK value. The parameter $\beta$ in (13) is set to 1 and the result is shown in Figure 12. It is clear from this figure that the second bandpass filtering signal contains most part of the impulsive signals and its kurtosis is the biggest in all the subband signals. Therefore, the impulsive signal with a very strong noise is detected by the proposed algorithm.

To test antinoise ability of the proposed method, we consider the signals used in Figure 7 . The result of the proposed method, compared with kurtosis of the original signal, is shown in Figure 13 (some of the kurtosis values are listed in Table 1). This figure shows that the kurtosis value calculated by the proposed method is bigger than kurtosis of the original signals except that it is slightly smaller in the region of SNR between 4 and 6. Especially in the strong noise cases, we can see from this figure that the proposed method can get a big kurtosis value. If it is assumed that the impulsive signal is detected when the kurtosis value is bigger than 4 , we then get that the proposed method can work when SNR is as low as -17 .

\section{Conclusion}

We have analyzed the factors that have an influence on the kurtosis value. Through some simulated analysis, we got that amplitude, not the frequency of signals, and noise make major contribution to kurtosis values. Then, we proposed an adaptive SK method. The core step of the proposed SK method is to find local maximum points, add window around maxima, merge windows in the frequency domain, and then filter signals according to the merged windows in the time domain. Unlike the conventional SK methods based on FFT, we use maximum points in spectrum to find out the proper bandwidth of filters to filter signals into subbands. The parameters of the proposed SK method are varying adaptively with different signals. Testing on a periodic impulsive signal with different SNR shows that the proposed method can detect the period impulsive signal for the SNR that is as low as -17 .

\section{Conflicts of Interest}

The authors declare that there are no conflicts of interest regarding the publication of this manuscript.

\section{Acknowledgments}

This work was supported in part by the National Natural Science Foundation of China (NSFC) (no. 61401305) and the 

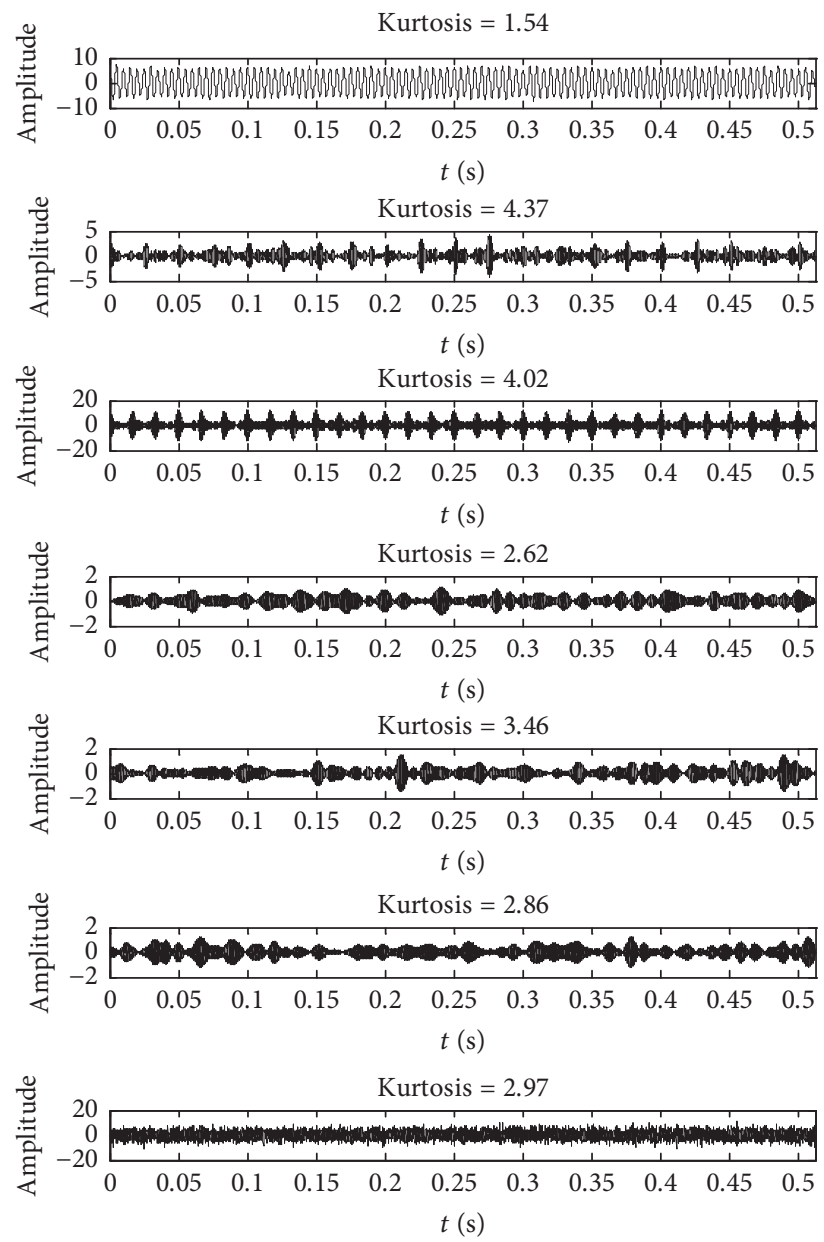

(a)
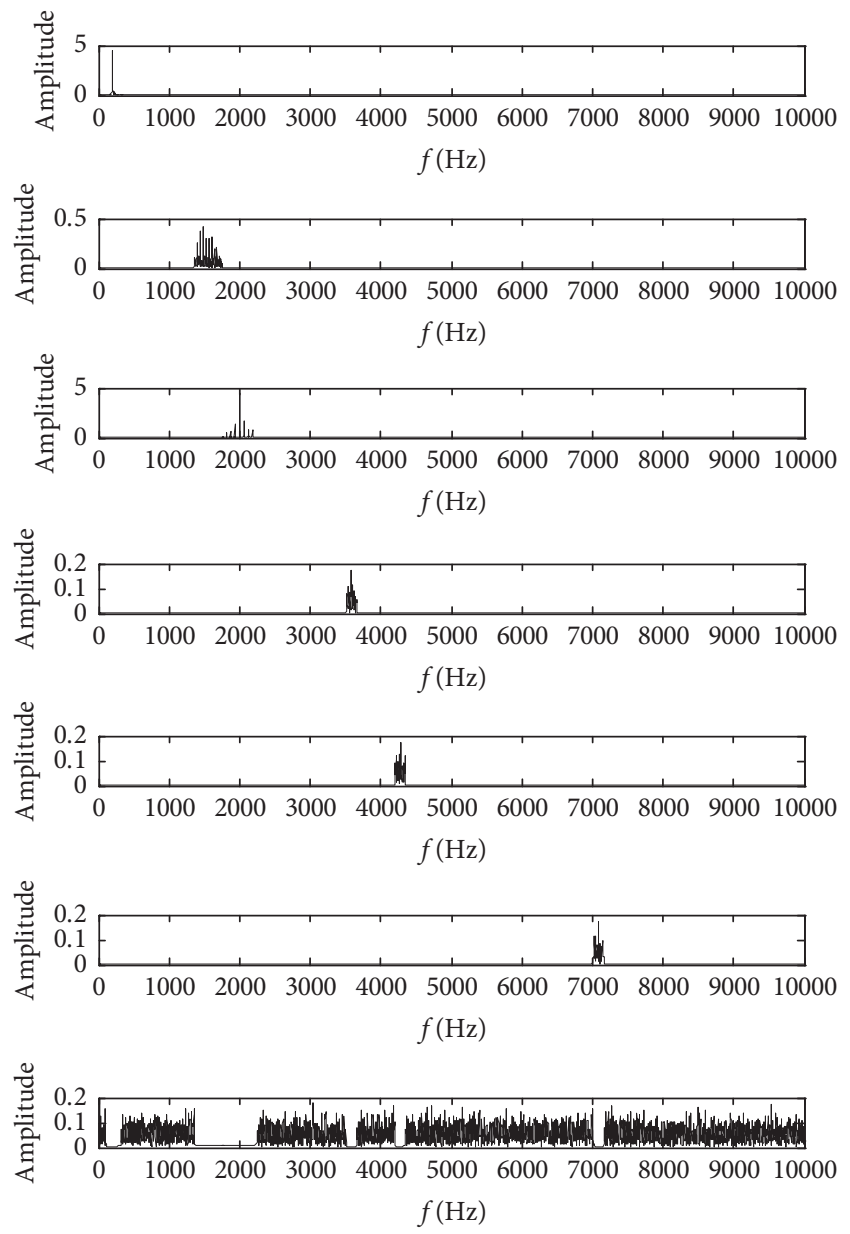

(b)

FIgURE 12: The result of the proposed method for the signal when SNR is -10 . (a) is bandpass filtering signals, and the bottom of (a) is the surplus signal. (b) is the spectrum of corresponding (a).

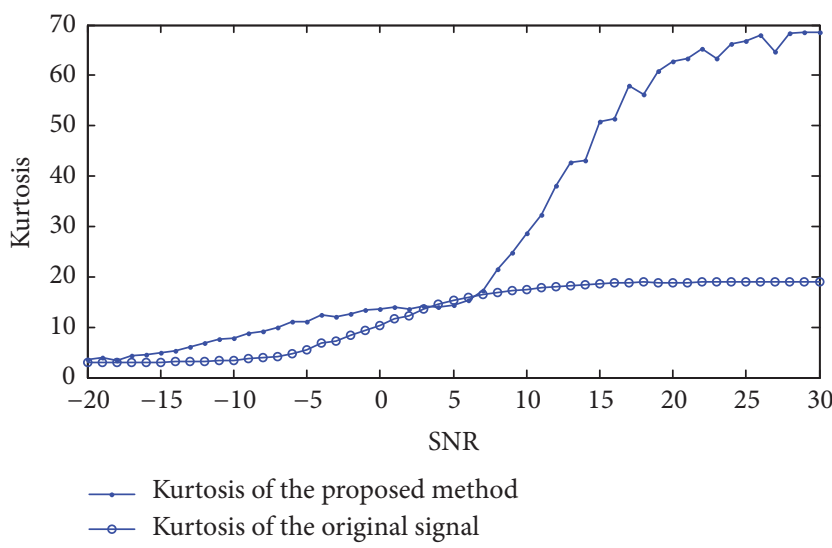

FIGURE 13: The result of the proposed method for signals with different SNR.

Natural Science Foundation of Tianjin, China (no. 15JCYBJC16500).

\section{References}

[1] D. Dyer and R. M. Stewart, "Detection of Rolling Element Bearing Damage by Statistical Vibration Analysis," Journal of Mechanical Design, vol. 100, no. 2, p. 229, 1978.

[2] R. F. Dwyer, "Detection of non-Gaussian signals by frequency domain kurtosis estimation," in Proceedings of the IEEE International Conference on Acoustics, Speech and Signal Processing (ICASSP '83), pp. 607-610, Boston, Mass, USA, 1984.

[3] J. Antoni, “The spectral kurtosis: a useful tool for characterising non-stationary signals," Mechanical Systems and Signal Processing, vol. 20, no. 2, pp. 282-307, 2006.

[4] J. Antoni and R. B. Randall, "The spectral kurtosis: application to the vibratory surveillance and diagnostics of rotating machines," Mechanical Systems and Signal Processing, vol. 20, no. 2, pp. 308-331, 2006.

[5] F. Jia, Y. Lei, H. Shan, and J. Lin, "Early fault diagnosis of bearings using an improved spectral kurtosis by maximum correlated kurtosis deconvolution," Sensors, vol. 15, no. 11, pp. 29363-29377, 2015. 
[6] Y. Li, L. Wang, and J. Guan, "A Spectrum Detection Approach for Bearing Fault Signal Based on Spectral Kurtosis," Shock and Vibration, vol. 2017, pp. 1-9, 2017.

[7] J. Xiang, Y. Zhong, and H. Gao, "Rolling element bearing fault detection using PPCA and spectral kurtosis," Measurement, vol. 75, pp. 180-191, 2015.

[8] T. Barszcz and A. JabŁoński, "A novel method for the optimal band selection for vibration signal demodulation and comparison with the Kurtogram," Mechanical Systems and Signal Processing, vol. 25, no. 1, pp. 431-451, 2011.

[9] Y. Wang and M. Liang, "An adaptive SK technique and its application for fault detection of rolling element bearings," Mechanical Systems and Signal Processing, vol. 25, no. 5, pp. 1750-1764, 2011.

[10] X. Zhang, J. Kang, L. Xiao, J. Zhao, and H. Teng, "A new improved Kurtogram and its application to bearing fault diagnosis," Shock and Vibration, vol. 2015, Article ID 385412, 22 pages, 2015.

[11] Y. Wang and M. Liang, "Identification of multiple transient faults based on the adaptive spectral kurtosis method," Journal of Sound and Vibration, vol. 331, no. 2, pp. 470-486, 2012.

[12] H. Liu, W. Huang, S. Wang, and Z. Zhu, "Adaptive spectral kurtosis filtering based on Morlet wavelet and its application for signal transients detection," Signal Processing, vol. 96, pp. 118124, 2014.

[13] J. S. Luo, D. J. Yu, and M. Liang, "A kurtosis-guided adaptive demodulation technique for bearing fault detection based on tunable-Q wavelet transform," Measurement Science and Technology, vol. 24, no. 5, Article ID 055009, 2013.

[14] W. A. Smith, Z. Fan, Z. Peng, H. Li, and R. B. Randall, "Optimised Spectral Kurtosis for bearing diagnostics under electromagnetic interference," Mechanical Systems and Signal Processing, 2015.

[15] Y. Yanli and D. Jiahao, "Analysis on frequency resolution of EMD based on B-spline interpolation," $A E \ddot{U}$ - International Journal of Electronics and Communications, 2015.

[16] Y. Yang, "A Signal Theoretic Approach for Envelope Analysis of Real-Valued Signals,” IEEE Access, vol. 5, pp. 5623-5630, 2017.

[17] X. Wang and K. Turitsyn, "Data-Driven Diagnostics of Mechanism and Source of Sustained Oscillations," IEEE Transactions on Power Systems, vol. 31, no. 5, pp. 4036-4046, 2016.

[18] D. Ho and R. B. Randall, "Optimisation of bearing diagnostic techniques using simulated and actual bearing fault signals," Mechanical Systems and Signal Processing, vol. 14, no. 5, pp. 763788, 2000. 


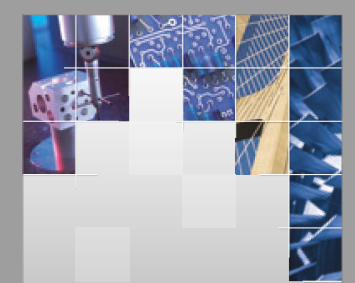

\section{Enfincering}
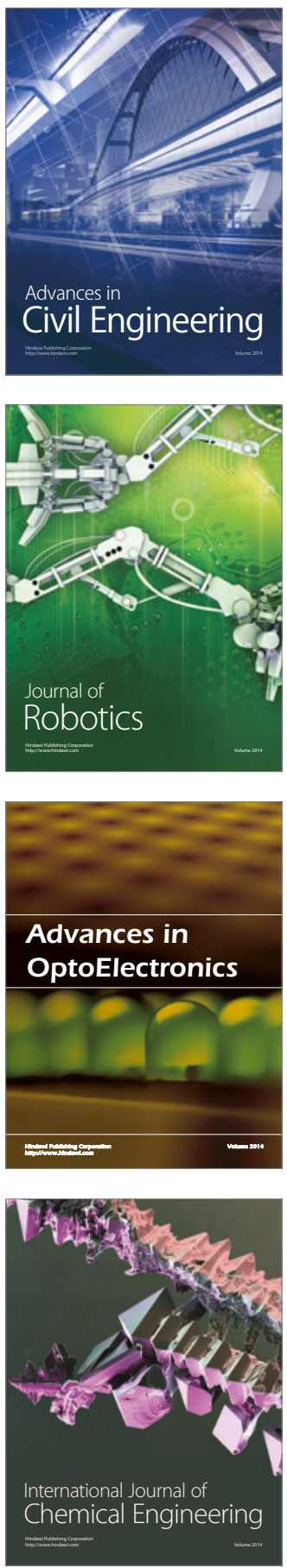

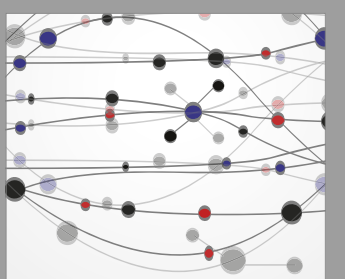

The Scientific World Journal

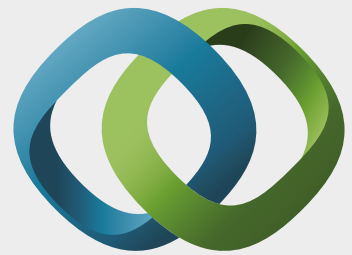

\section{Hindawi}

Submit your manuscripts at

https://www.hindawi.com
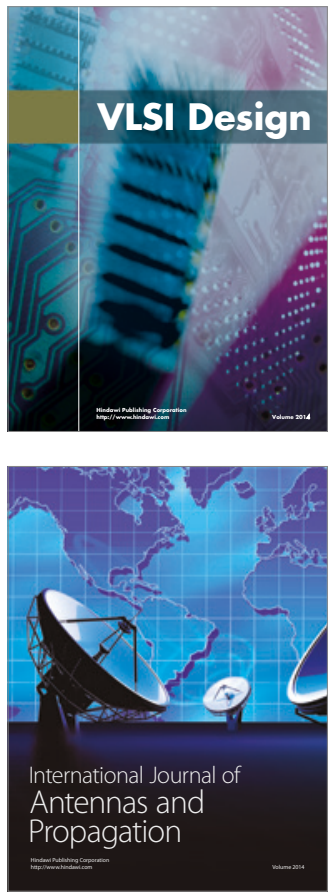

\section{Rotating}

Machinery
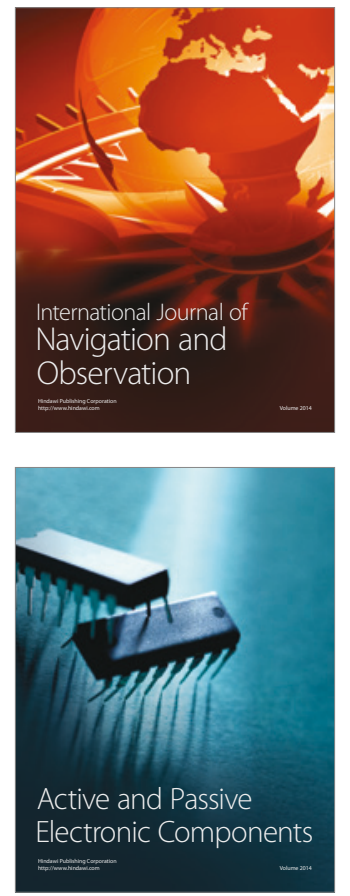
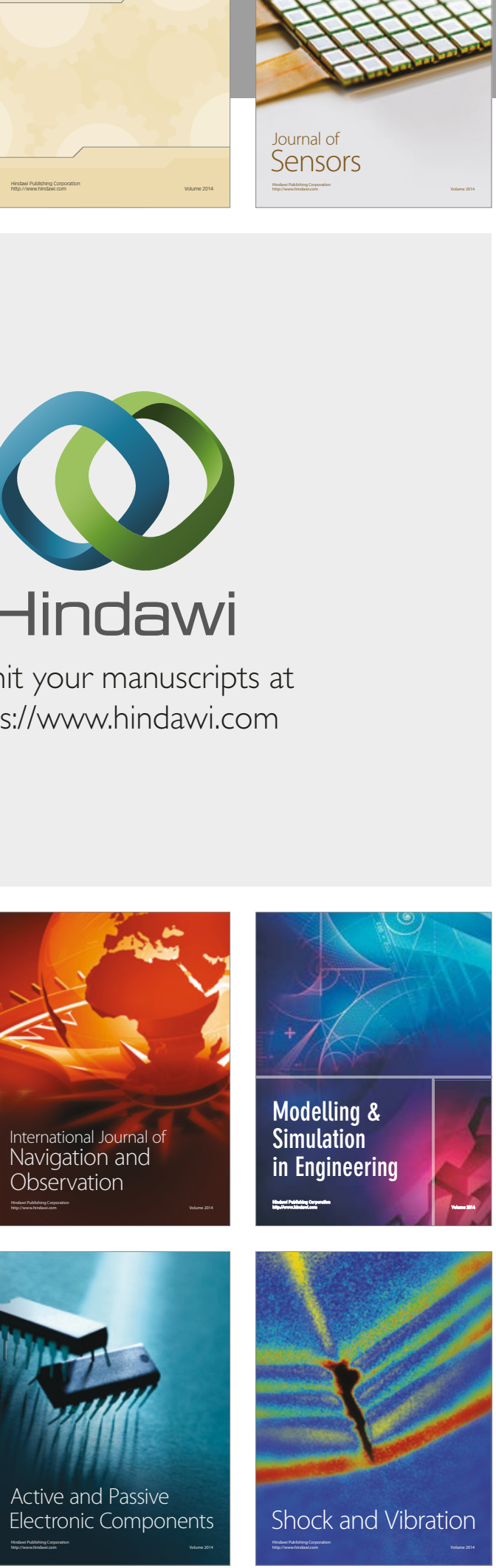
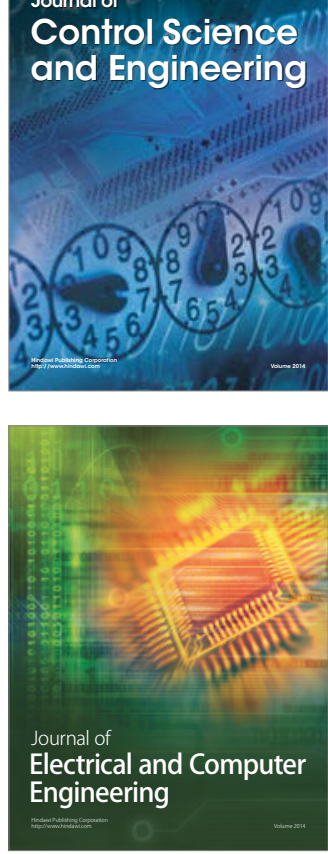

Distributed

Journal of

Control Science

and Engineering
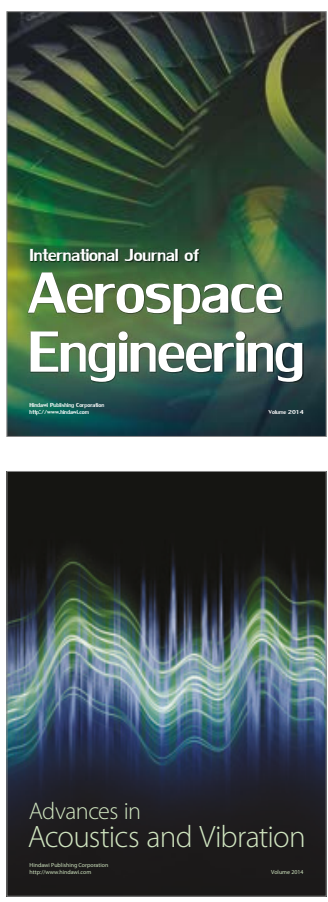

Sensor Networks 